\title{
The relationship between personality disorders and mental health, substance use severity and quality of life among injecting drug users
}

\author{
Tania M Gibbie, Leanne M Hides, Sue M Cotton, Daniel I Lubman, Campbell Aitken and Margaret Hellard
}

T he diagnosis of personality disorder (PD) encompasses a broad range of personality traits (patterns of perceiving, thinking about, relating to and interacting with people) that are inflexible, maladaptive, and cause significant distress and impairment without treatment. ${ }^{1-2}$ PDs are stable over time and result in interpersonal difficulties across multiple settings. ${ }^{2}$ Based on their characteristics, PDs can be grouped into three clusters: Cluster A, where individuals appear odd or eccentric; Cluster $\mathrm{B}$, where individuals appear dramatic, emotional or erratic; and Cluster C, where individuals appear anxious or fearful. ${ }^{2}$

According to the 1997 National Survey of Mental Health and Wellbeing (NSMHW), about $6.5 \%$ of the adult Australian population has symptoms consistent with one or more PDs. Factors identified as associated with PD include being younger than 25 years, male, single, having a concurrent affective, anxiety or substance use disorder, or a physical condition. ${ }^{3}$ Individuals with PDs are more likely to have greater mental and physical health problems and more frequent visits to general practitioners, psychiatrists and psychologists than those without the diagnosis. ${ }^{3}$ They also report a greater number of chronic physical conditions (eg, asthma, cancer). ${ }^{3}$

Several studies estimate up to $80 \%$ of injecting drug users (IDUs) have a lifetime history of mental disorder, with mood, anxiety and Cluster B PDs (dramatic, emotional or erratic disorders; eg, borderline PD, antisocial PD) the most common. ${ }^{4}$ Most studies conducted within substance misuse treatment settings identify antisocial PD (about $30 \%-40 \%$ ) as the most common PD. ${ }^{5-9}$ An Australian study of heroin users entering treatment ${ }^{4}$ reported high rates of current major depression disorders (28\%), antisocial (72\%) and borderline (47\%) PDs. Similarly, another study ${ }^{10}$ reported that $45 \%$ of heroin users enrolled in the Australian Treatment Outcome Study were diagnosed with borderline PD, which was associated with higher levels of poor health care behaviour (including overdose, needle-related risk-taking and attempted suicide).

\section{ABSTRACT}

Objective: To determine the relationship between personality disorders (PDs) and substance use severity, mental health symptoms and disorders and quality of life ( $\mathrm{Q} \mathrm{L}$ ) among injecting drug users (IDUs).

Design, setting and participants: A cross-sectional study of 103 IDUs accessing a needle and syringe program and a primary health centre in Melbourne, Australia.

Main outcome measures: Presence of PDs was assessed using the International Personality Disorder Examination ICD-10 Screener. Axis I mental health disorders, psychological distress and QoL were also assessed.

Results: Ninety per cent of participants scored positive for one or more PD. Having a Cluster A or Cluster B PD was associated with greater severity of substance use. The presence of a current mental health disorder was associated with all types of PD except dissocial PD. Only Cluster C PDs were associated with self-reported levels of psychological distress. Cluster C PDs were more strongly associated with substance use, mental health and QoL variables than Cluster A or B, although the number of PDs present had the strongest associations with these variables.

Conclusions: IDUs had high rates of PD symptoms, which were associated with the presence of concurrent mental health disorders, more severe levels of psychological distress and substance use and low perceived QoL. IDUs require comprehensive models of care, including access to mental health practitioners with expertise in co-occurring disorders.

MJA 2011; 195: S16-S21

Comparatively few studies have investigated the prevalence of PDs in non-treatment settings, such as community-based needle and syringe programs (NSPs), or the impact of PDs other than those in Cluster B. One study conducted in a community-based NSP in the United States reported that 37\% of clients had antisocial PD; however, this was the only type of PD assessed. ${ }^{11}$ Similar studies are yet to be conducted within nontreatment settings in Australia.

Our study aimed to determine the relationship between the different clusters of PD and substance use severity, mental health symptoms/disorders, and quality of life (QoL) among IDUs accessing an NSP. We predicted Cluster B PDs would be most commonly identified, and would be associated with higher levels of substance use severity, psychological distress, increased mental health disorders, and poorer QoL.

\section{METHOD}

\section{Participants}

Participants were 103 people (23.2\% response rate) who visited an NSP and a primary health centre in Melbourne, Australia, between February and July 2004. Participants were eligible if they were over 16 years of age and able to give informed consent. The sample was predominantly male $(72.8 \%)$, with a mean age of 33.6 years (SD, 8.8; range, 18-67). The recruitment procedures, demographic and substance use characteristics of the sample have been described elsewhere. ${ }^{12}$

\section{Measures}

Personality disorders: PDs were assessed using the 59-item International Personality Disorder Examination (IPDE) ICD-10 Screener. 1,3 Participants respond "true" or "false" to each item — eg, "People think I'm cold and detached" (dissocial PD). Each question assesses one of the criteria for each of nine specific ICD-10 PDs. ${ }^{13}$ These ICD-10 PDs are grouped into three clusters:

- Cluster A - paranoid, schizoid PD;

- Cluster B - dissocial (DSM-IV antisocial PD), histrionic, emotionally unstable-impulsive $\mathrm{PD}$, impulsive and borderline subtypes (DSM-IV borderline PD); and 
- Cluster C - anankastic (DSM-IV obsessive-compulsive PD), anxious (DSM-IV avoidant), dependent PD. ${ }^{14}$

This screening tool was chosen for its brevity, ease of administration and ability to be compared with NSMHW data. ${ }^{3}$

Axis I mental health disorders: the Mini International Neuropsychiatric Interview $(\mathrm{MINI})^{15}$ was used to identify current Axis I diagnoses disorders. The MINI has high levels of reliability and good concordance with gold standard structured diagnostic measures. ${ }^{15,16}$

Psychological distress: the Kessler Psychological Distress Scale $(\mathrm{K} 10),{ }^{17}$ a 10 -item measure of general psychological distress, has been found to reliably predict the presence of a current depressive or anxiety disorder at a cut-off score of $\geqslant 17$ in an Australian community sample. ${ }^{18}$

Substance use: the Alcohol, Smoking and Substance Involvement Screening Test (ASSIST), version 2.1, ${ }^{19}$ is an eight-item measure of the frequency of substance use of 10 substances, as well as dependence symptoms and substance-related problems. The ASSIST has high levels of internal consistency, construct, concurrent and discriminant validity. ${ }^{20}$ A total substance involvement score (sum of response weights for items 1-8 across all substances) is derived. ${ }^{20}$

Quality of life: the World Health Organization Quality of Life (WHOQOL)-BREF contains 26 questions and focuses upon the respondents' perceived QoL. Assessment of QoL is reflected by four domains: physical, psychological, social and environmental. Transformed scores range from 0 to 100 , with higher scores reflecting higher perceptions of QoL. ${ }^{21}$

\section{Procedure}

Ethics approval was provided by the research and ethics committees of affiliated institutions. NSP clients were approached by the researcher or NSP staff and invited to participate. Once informed consent was obtained, participants were interviewed over a 1-hour period. Participants were reimbursed $\$ 10$ for travel-related expenses.

\section{Statistical analysis}

SPSS, version 16.0 (SPSS Inc, Chicago, Ill, USA) was used for data analysis. Simple descriptive summary statistics were used to describe the sample.

The independent variable was PD. PD was assessed by type, the number of positive
PDs, and cluster (eg, Cluster A: yes/no). Dependent variables were severity of substance use, presence of current Axis I mental health problem, psychological distress (K10 total score), and QoL total and subscale scores. To determine the relationship between PD and categorical dependent variables (eg, presence/absence of mental health disorder), logistic regressions were used. B coefficients, Wald $\chi^{2}$ and odds ratios are reported. For continuous dependent variables, standard regression was employed. B and standardised coefficients are reported. Significance was set at $P<0.05$.

Rather than entering all nine IPDE PDs into the regression models, univariate mod-

\section{Mini International Neuropsychiatric Interview (MINI) diagnosis, personality disorder, substance use, psychological distress and quality of life in 103 injecting drug users}

\begin{tabular}{ll} 
Variable & No. \\
\hline MINI diagnosis & $51(49.5 \%)$ \\
Mood disorder & $56(54.4 \%)$ \\
Anxiety disorder & $10(9.7 \%)$ \\
Psychotic disorder & \\
Personality disorder & $73(70.9 \%)$ \\
Cluster A & $55(53.4 \%)$ \\
Paranoid & $50(48.5 \%)$ \\
Schizoid & $74(71.8 \%)$ \\
Cluster B & $35(34.0 \%)$ \\
Dissocial & $55(53.4 \%)$ \\
Impulsive & $59(57.3 \%)$ \\
Borderline & $17(16.5 \%)$ \\
Histrionic & $65(63.1 \%)$ \\
Cluster C & $42(40.8 \%)$ \\
Anankastic & $47(45.6 \%)$ \\
Anxious & $37(35.9 \%)$ \\
Dependent & Mean (SD) \\
\cline { 2 - 2 } Severity of substance use* & $48.9(15.1)$ \\
Psychological distress & \\
Quality of life & $28.5(8.9)$ \\
Physical & $12.3(2.9)$ \\
Social & $12.1(3.3)$ \\
Emotional & $11.8(3.5)$ \\
\hline Alcohol & $12.2(3.1)$ \\
\hline
\end{tabular}

*Alcohol, Smoking and Substance Involvement Screening Test total score. † Kessler Psychological Distress Scale total score. $¥$ World Health Organization Quality of Life (WHOQOL)-BREF domain scores. els were tested with only one PD (eg, dissocial: yes/no) in the model. This approach was adopted to avoid the problem of multicollinearity due to overlap between PD symptoms. Similarly, number of PDs was entered as a sole predictor in the regression models. In order to determine which PD cluster best predicted the dependent variables, all three cluster variables (ie, Cluster A: yes/no; Cluster B: yes/no; Cluster C: yes/no) were entered into the models.

\section{RESULTS}

\section{Sample characteristics}

All 103 participants had a current substance use disorder and 25.2\% (26) had a concurrent alcohol use disorder. Almost all participants (95.0\%) were daily tobacco users and 39.8\% (41) were daily cannabis users. Sixty-three per cent (65) reported daily or almost daily opiate use with $19.4 \%$ (20) reporting weekly use. Ten participants (13\%) used amphetamines weekly, and weekly sedative use was reported by a third of the participants (33). ${ }^{11}$

Almost $70 \%$ of participants (72) had a current Axis I mental health disorder (Box 1 ), with $57 \%$ (60) having more than one disorder.

\section{Rates of PDs}

Ninety per cent of participants (93) scored positive for one or more $\mathrm{PD}$. Of these, $13.6 \%$ (14) had one PD, 15.5\% (16) had two PDs, and $61.2 \%$ (63) had three or more PDs. The mean number of PDs was 3.9 (SD, 2.6). There were no differences by sex with respect to type or number of PDs. Borderline symptoms were the most common type of PD symptoms reported, followed by impulsive and paranoid PD symptoms, and then schizoid and anxious PD symptoms. Histrionic symptoms were the least common type (Box 1).

\section{Severity of substance use}

Standard regression analyses were conducted to determine the relationship between PD symptoms and severity of substance use (ASSIST total score) (Box 2). Borderline, impulsive and paranoid PD symptoms were associated with more severe substance use. Histrionic PD symptoms were unrelated to severity of substance use. There was a strong association between the number of PDs and severity of substance use. Having a Cluster A or Cluster B PD was associated with greater severity of substance use. 


\section{Psychological distress}

Standard regression analyses (Box 2) indicated that anxious PD symptoms had the strongest correlation with K10 total scores. The number of PDs was moderately associated with psychological distress, and Cluster C PDs were associated with psychological distress.

\section{Mental health disorders}

Logistic regression analyses revealed that the presence of a current Axis I mental health disorder was associated with increased risk of all types of PDs with the exception of dissocial PD (Box 3). Individuals with anxious PD were nearly 11 times more likely to have a mental health disorder. The number of PDs also significantly increased the chances of having a mental health disorder. Scoring positive for a Cluster A or Cluster C PD was predictive of having an Axis I mental health disorder.

\section{Quality of life}

Associations between PDs and QoL domains are shown in Box 4. Borderline, anxious and dependent PD symptoms had the highest positive correlations with the physical domain of the WHOQOL-BREF. Only Cluster $C$ was positively associated with physical QoL. Poorer psychological QoL was associated with anxious, borderline, dependent and schizoid PD, and with scoring positive for a Cluster B or Cluster C PD. Poorer social QoL was associated with scoring positive for schizoid PD symptoms and a Cluster A PD. Environmental QoL was associated with scoring positive for dependent, anxious and borderline PD symptoms and Cluster C PDs. The number of PDs was associated with poorer outcomes across all QoL domains.

\section{DISCUSSION}

Our study is the first to comprehensively examine rates of Cluster A, B and C PDs among IDUs accessing a community-based NSP in Australia. We report high levels of PD symptoms, with $90 \%$ of participants scoring positive for one or more PD; nearly 14 times higher than Australia's general population prevalence of $6.5 \%$ (ascertained using an identical instrument). ${ }^{3}$ The most prevalent $\mathrm{PD}$ in this study was borderline PD (57.3\%), which again was dramatically higher than the $0.96 \%$ identified in the Australian general population ${ }^{3}$ and higher than that found in past research, ${ }^{5-9}$ including the findings for heroin users in the

2 Association between personality disorder (PD) and severity of substance use
and psychological distress $B$

\begin{tabular}{|c|c|c|c|c|c|}
\hline & $B$ & SE & $\beta$ & $t$ & $P$ \\
\hline \multicolumn{6}{|c|}{ Severity of substance use } \\
\hline \multicolumn{6}{|l|}{ Type of PD* } \\
\hline Paranoid & 12.36 & 2.00 & 0.41 & 4.53 & $<0.001$ \\
\hline Schizoid & 6.50 & 2.92 & 0.22 & 2.23 & 0.028 \\
\hline Dissocial & 6.74 & 3.08 & 0.21 & 2.19 & 0.031 \\
\hline Impulsive & 13.42 & 2.68 & 0.45 & 5.01 & $<0.001$ \\
\hline Borderline & 14.65 & 2.64 & 0.48 & 5.54 & $<0.001$ \\
\hline Histrionic & 6.40 & 4.00 & 0.16 & 1.61 & 0.110 \\
\hline Anankastic & 6.78 & 2.96 & 0.22 & 2.29 & 0.024 \\
\hline Anxious & 9.32 & 2.85 & 0.31 & 3.26 & 0.001 \\
\hline Dependent & 12.10 & 2.87 & 0.39 & 4.22 & $<0.001$ \\
\hline Number of PDs ${ }^{\dagger}$ & 3.15 & 0.49 & 0.54 & 6.42 & $<0.001$ \\
\hline \multicolumn{6}{|l|}{ PD clusters ${ }^{\ddagger}$} \\
\hline Cluster A & 7.00 & 3.23 & 0.21 & 2.16 & 0.033 \\
\hline Cluster B & 9.26 & 3.10 & 0.27 & 2.99 & 0.004 \\
\hline Cluster C & 5.51 & 3.17 & 0.18 & 1.73 & 0.085 \\
\hline
\end{tabular}

Psychological distress

Type of PD $\$$

\begin{tabular}{lllllr} 
Paranoid & 4.86 & 1.70 & 0.27 & 2.90 & 0.005 \\
Schizoid & 6.23 & 1.65 & 0.35 & 3.78 & $<0.001$ \\
Dissocial & 4.00 & 1.82 & 0.21 & 2.20 & 0.030 \\
Impulsive & 5.72 & 1.67 & 0.32 & 3.43 & 0.001 \\
Borderline & 7.44 & 1.62 & 0.42 & 4.60 & $<0.001$ \\
Histrionic & 5.63 & 2.30 & 0.24 & 2.44 & 0.016 \\
Anankastic & 4.33 & 1.74 & 0.24 & 2.49 & 0.014 \\
Anxious & 9.31 & 1.51 & 0.52 & 6.19 & $<0.001$ \\
Dependent & 7.39 & 1.68 & 0.40 & 4.40 & $<0.001$ \\
Number of PDs & 1.93 & 0.28 & 0.56 & 6.81 & $<0.001$ \\
PD clusters** & & & & & 0.113 \\
Cluster A & 3.01 & 1.88 & 0.16 & 1.60 & 0.060 \\
Cluster B & 3.40 & 1.81 & 0.17 & 1.88 & 0.002 \\
Cluster C & 6.03 & 1.85 & 0.33 & 3.26 & \\
\hline
\end{tabular}

* Based on a series of standard regression models with each type of PD as the predictor of Alcohol, Smoking and Substance Involvement Screening Test (ASSIST) total score; $\beta$ is equivalent to point-biserial correlation between PD and ASSIST total. † Based on a standard regression model with number of PDs as a predictor of substance use severity; $\beta$ is equivalent to Pearson's product moment correlation between number of PDs and ASSIST total. ¥Three clusters were included in a standard regression model as predictors of substance use severity. §Based on a series of standard regression models with each type of PD as the predictor of Kessler Psychological Distress Scale (K10) total score; $\beta$ is equivalent to point-biserial correlation between PD and K10 total. ๆ Based on a standard regression model with number of PDs as a predictor psychological distress; $\beta$ is equivalent to Pearson's product moment correlation between number of PDs and K10 total score. ${ }^{\star *}$ Three clusters were included in a standard regression model as predictors of psychological distress.

Australian Treatment Outcome Study $(45 \%){ }^{10}$

The prevalence of mood disorders (49.5\%) identified in our study appears much higher than that among heroin users entering treatment (26\%-33\% with major depression). ${ }^{4}$ This suggests that IDUs who access NSPs have higher rates of Axis I mental health disorders than those who enter treatment, although the study by Ross and colleagues used a different measure to assess for mental heath disorders.

Consistent with previous research, Cluster B PDs were the most prevalent. ${ }^{4-11}$ The rate of 


\begin{tabular}{|c|c|c|c|c|c|c|}
\hline & $B$ & SE & Wald $\chi^{2}$ & $P$ & OR & $95 \% \mathrm{Cl}$ \\
\hline \multicolumn{7}{|l|}{ Type of PD* } \\
\hline Paranoid & 1.55 & 0.47 & 11.08 & 0.001 & 4.70 & $1.89-11.70$ \\
\hline Schizoid & 1.04 & 0.45 & 5.37 & 0.020 & 2.84 & $1.18-6.86$ \\
\hline Dissocial & 0.18 & 0.46 & 0.15 & 0.695 & 1.20 & $0.49-2.92$ \\
\hline Impulsive & 1.14 & 0.45 & 6.50 & 0.011 & 3.11 & $1.30-7.45$ \\
\hline Borderline & 1.38 & 0.45 & 9.42 & 0.002 & 3.98 & $1.65-9.63$ \\
\hline Histrionic & 2.20 & 1.06 & 4.35 & 0.037 & 9.02 & $1.14-71.30$ \\
\hline Anankastic & 1.01 & 0.47 & 4.60 & 0.032 & 2.76 & $1.09-6.96$ \\
\hline Anxious & 2.38 & 0.59 & 16.36 & $<0.001$ & 10.75 & $3.40-33.97$ \\
\hline Dependent & 1.49 & 0.54 & 7.54 & 0.006 & 4.43 & $1.53-12.82$ \\
\hline Number of PDs ${ }^{\dagger}$ & 0.46 & 0.11 & 16.88 & $<0.001$ & 1.58 & $1.27-1.97$ \\
\hline \multicolumn{7}{|l|}{ PD clusters ${ }^{\ddagger}$} \\
\hline Cluster A & 1.07 & 0.52 & 4.21 & 0.040 & 2.90 & $1.05-8.03$ \\
\hline Cluster B & 0.31 & 0.53 & 0.34 & 0.559 & 1.36 & $0.48-3.83$ \\
\hline Cluster C & 1.33 & 0.52 & 6.52 & 0.011 & 3.79 & $1.36-10.54$ \\
\hline
\end{tabular}

* Based on a series of logistic regression models with each type of PD as the only predictor of mental disorder (yes/no). † Based on a logistic regression model with number of PDs as a predictor of presence of mental disorder. $¥$ Three clusters were included in a logistic regression model as predictors of presence of mental health disorder.

dissocial PD was lower than expected, although this may be associated with the absence of one of the six ICD-10 criteria for dissocial PD from the IPDE screener used in our study. ${ }^{14}$ Little is known about the rates of other ICD-10 PDs in populations of IDUs, but current results document high rates of comorbid PDs in this population.

Cluster A PDs were associated with substance use severity, presence of an Axis I mental health disorder and poorer social QoL. Cluster B PDs were associated with increased substance use severity, whereas borderline PD symptoms were specifically associated with poorer QoL on psychological and environmental domains. Overall, Cluster C PDs had the strongest associations, including severity of psychological distress, substance use, presence of an Axis I mental health disorder, and lower QoL on the physical, psychological and environmental domains. These results are inconsistent with our predictions and previous research showing that these variables would have the strongest associations with borderline PDs. While these findings may indicate the presence of a Cluster C PD, with anxious PD being associated with the worst outcomes, this is the first study to comprehensively examine rates and outcomes of Cluster A, B and C PDs in this population and replication is needed. Nonetheless, although Cluster B PDs may present more overt challenges to services, Cluster C symptoms may particularly need to be targeted within substance use treatment settings.

The number of PDs was also predictive of higher levels of psychological distress, more severe substance use problems and lower QoL (across all domains). Similar to other researchers, we found that individuals with a PD suffer significant impairment across both mental health and physical conditions. This indicates that the observed effects of various personality symptoms may relate more to the overall severity of PD symptoms rather than individual differences between PDs. These findings are consistent with recent suggestions that a global dimensional measure of the likelihood of PD may prove to be equally useful to, or even more useful than, individual diagnoses of PDs. ${ }^{14}$

Several limitations of our study must be considered in interpreting these results. First, the IPDE screener is a screening tool, not a diagnostic measure, so it is likely the prevalence of actual PD within this sample is overestimated. ${ }^{3}$ However, previous studies have used a combination of diagnostic interviews and/or screening tools and our findings are generally consistent with these studies. ${ }^{3-8}$ Second, high levels of Axis I mental health disorders and psychological distress were reported in this group. It is likely that items on the IPDE screener may be associated with longstanding symptoms of psychological distress. This may also account for the higher rates of PDs identified here; however, the opposite relationship may be true. Third, it was not possible to determine the effect of recent substance use on the level of psychological symptoms reported, which may have affected the reliability of self-reported PD symptoms. Fourth, reliance on self-report measures, the single assessment period, low response rate, and not recruiting a pure sample of nontreatment seekers (ie, some individuals may be receiving treatment as well as accessing the NSP) may have affected our results. For example, the low prevalence of dissocial PD identified may be due to the low response rate. Further research with greater sample sizes is required to tease out these relationships. Finally, screening tools have been criticised for being more sensitive to state rather than trait effects. ${ }^{14}$ In this regard, future studies should incorporate more structured diagnostic assessments, the collection of relevant collateral information, as well as longitudinal follow-up to determine the stability of the PD symptoms identified.

In summary, IDUs accessing a community-based NSP in Australia reported high rates of $\mathrm{PD}$ symptoms, with individuals who endorsed more PD symptoms suffering higher levels of psychological distress, increased substance use severity and lower QoL. While PDs may be associated with these variables, the high rate of $\mathrm{PD}$ symptoms suggests that this population has significant mental health issues and requires a comprehensive package of care. Consideration of the impact of Cluster C symptoms on outcomes is also important, especially as these symptoms are typically not addressed within drug treatment programs. As such, the findings highlight the need to develop more holistic models of treatment within NSP settings, including access to mental health practitioners with expertise in managing personality and co-occurring disorders.

\section{ACKNOWLEDGEMENTS}

We thank the clients for their participation and the staff at HealthWorks Primary Health Care Centre, in particular Jacqui Brown for her support of this study. Thanks to Harriet Devlin and Lisa Catania for their assistance with participant recruitment. Our study was completed with the assistance of funding from Perpetual Trustees, the Flora and Frank Leith Charitable Trust, the Jack Brockhoff Foundation, the Grosvenor Settlement, the Sunshine Foundation and Danks Trust. Leanne Hides and Dan Lubman are supported by the Colonial Foundation. Margaret Hellard is supported by a National Health and 
4 Association between personality disorder (PD) and quality of life

\begin{tabular}{|c|c|c|c|c|c|c|c|c|c|c|c|}
\hline $\begin{array}{l}\text { Quality of life } \\
\text { domain }\end{array}$ & $B$ & SE & $\beta$ & $t$ & $P$ & $\begin{array}{l}\text { Quality of life } \\
\text { domain }\end{array}$ & $B$ & SE & $\beta$ & $t$ & $P$ \\
\hline Physical & & & & & & Social & & & & & \\
\hline Type of PD* & & & & & & Type of PD* & & & & & \\
\hline Paranoid & -0.98 & 0.58 & -0.17 & -1.70 & 0.093 & Paranoid & -1.38 & 0.69 & -0.20 & -2.00 & 0.048 \\
\hline Schizoid & -1.71 & 0.58 & -0.29 & -3.06 & 0.003 & Schizoid & -2.33 & 0.66 & -0.34 & -3.53 & 0.001 \\
\hline Dissocial & -1.36 & 0.61 & -0.22 & -2.34 & 0.028 & Dissocial & -0.67 & 0.74 & -0.09 & -0.90 & 0.372 \\
\hline Impulsive & -1.60 & 0.56 & -0.28 & -2.85 & 0.005 & Impulsive & -0.27 & 0.70 & -0.04 & -0.39 & 0.698 \\
\hline Borderline & -2.07 & 0.55 & -0.36 & -3.77 & $<0.001$ & Borderline & -1.00 & 0.70 & -0.14 & -1.43 & 0.155 \\
\hline Histrionic & -0.89 & 0.79 & -0.11 & -1.12 & 0.268 & Histrionic & 0.52 & 0.96 & 0.05 & 0.54 & 0.590 \\
\hline Anankastic & -1.38 & 0.58 & -0.23 & -2.39 & 0.019 & Anankastic & -0.82 & 0.71 & -0.12 & -1.15 & 0.253 \\
\hline Anxious & -1.84 & 0.56 & -0.32 & -3.32 & 0.001 & Anxious & -1.18 & 0.69 & -0.17 & -1.71 & 0.091 \\
\hline Dependent & -1.89 & 0.58 & -0.31 & -3.21 & 0.002 & Dependent & -1.52 & 0.72 & -0.21 & -2.12 & 0.037 \\
\hline Number of PDs ${ }^{\dagger}$ & -0.50 & 0.10 & -0.44 & -4.82 & $<0.001$ & Number of PDs ${ }^{\dagger}$ & -0.33 & 0.13 & -0.24 & -2.50 & 0.014 \\
\hline PD clusters ${ }^{\ddagger}$ & & & & & & PD clusters ${ }^{\ddagger}$ & & & & & \\
\hline Cluster A & -1.00 & 0.64 & -0.16 & -1.57 & 0.120 & Cluster A & -1.85 & 0.83 & -0.24 & -2.24 & 0.028 \\
\hline Cluster B & -1.09 & 0.61 & -0.17 & -1.78 & 0.079 & Cluster B & 0.36 & 0.80 & 0.05 & 0.46 & 0.650 \\
\hline Cluster C & -1.76 & 0.62 & -0.29 & -2.82 & 0.006 & Cluster C & -0.66 & 0.81 & -0.09 & -0.81 & 0.420 \\
\hline Psychological & & & & & & Environmental & & & & & \\
\hline Type of PD* & & & & & & Type of PD* & & & & & \\
\hline Paranoid & -1.53 & 0.65 & -0.23 & -2.36 & 0.020 & Paranoid & -1.61 & 0.61 & -0.26 & -2.66 & 0.009 \\
\hline Schizoid & -2.29 & 0.62 & -0.35 & -3.69 & $<0.001$ & Schizoid & -1.44 & 0.61 & -0.23 & -2.37 & 0.020 \\
\hline Dissocial & -1.45 & 0.69 & -0.21 & -2.10 & 0.038 & Dissocial & -1.76 & 0.65 & -0.27 & -2.72 & 0.008 \\
\hline Impulsive & -1.76 & 0.64 & -0.27 & -2.75 & 0.007 & Impulsive & -1.64 & 0.61 & -0.26 & -2.71 & 0.008 \\
\hline Borderline & -2.52 & 0.62 & -0.38 & -4.08 & $<0.001$ & Borderline & -1.94 & 0.60 & -0.31 & -3.24 & 0.002 \\
\hline Histrionic & -1.45 & 0.90 & -0.16 & -1.63 & 0.107 & Histrionic & -1.09 & 0.85 & -0.13 & -1.29 & 0.201 \\
\hline Anankastic & -1.60 & 0.66 & -0.24 & -2.25 & 0.016 & Anankastic & -1.09 & 0.63 & -0.17 & -1.73 & 0.087 \\
\hline Anxious & -3.62 & 0.56 & -0.55 & -6.49 & $<0.001$ & Anxious & -1.95 & 0.60 & -0.31 & -3.27 & 0.001 \\
\hline Dependent & -2.57 & 0.64 & -0.37 & -4.01 & $<0.001$ & Dependent & -2.29 & 0.62 & -0.35 & -3.72 & $<0.001$ \\
\hline Number of PDs ${ }^{\dagger}$ & & & & & & Number of PDs ${ }^{\dagger}$ & -0.54 & 0.11 & -0.44 & -4.84 & $<0.001$ \\
\hline PD clusters ${ }^{\ddagger}$ & -0.68 & 0.11 & -0.53 & -6.14 & $<0.001$ & PD clusters ${ }^{\ddagger}$ & & & & & \\
\hline Cluster A & -1.24 & 0.68 & -0.17 & -1.83 & 0.071 & Cluster A & -1.01 & 0.72 & -0.15 & -1.42 & 0.160 \\
\hline Cluster B & -1.36 & 0.65 & -0.19 & -2.08 & 0.040 & Cluster B & -0.72 & 0.69 & -0.11 & -1.05 & 0.298 \\
\hline Cluster C & -2.50 & 0.67 & -0.37 & -3.75 & $<0.001$ & Cluster C & -1.50 & 0.70 & -0.23 & -2.13 & 0.036 \\
\hline
\end{tabular}

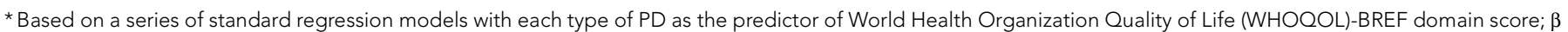

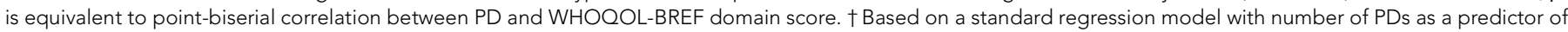

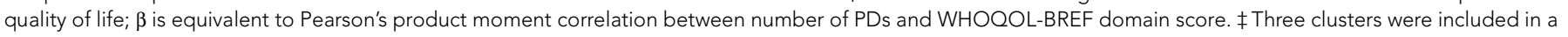
standard regression model as predictors of quality of life.

Medical Research Council Career Development Award and a VicHealth Fellowship.

\section{COMPETING INTERESTS}

None relevant to this article declared (ICJME disclosure forms completed).

\section{AUTHOR DETAILS}

Tania M Gibbie, BBSc(Hons), MPsych(Health), Research Fellow and Psychologist ${ }^{1,2}$
Leanne Hides, BBehSc(Hons), PhD(Clin), Senior Research Fellow ${ }^{3}$

Sue M Cotton, BBSc(Hons), MAppSc(Statistics), $\mathrm{PhD}$, Ronald Phillip Griffith Fellow and Senior Research Fellow ${ }^{4}$

Dan I Lubman, PhD, FRANZCP, FAChAM,

Clinical Director, and Professor of Addiction Studies and Services $2,5,6$

Campbell Aitken, BSc(Hons), PhD, Senior Research Fellow ${ }^{7}$

Margaret Hellard, PhD, FRACP, FAFPHM, Director ${ }^{7}$

1 Infectious Diseases Unit, The Alfred Hospital, Melbourne, VIC.
2 Monash University, Melbourne, VIC.

3 Institute of Health and Biomedical Innovation, Queensland University of Technology, Brisbane, QLD.

4 Orygen Youth Health Research Centre, University of Melbourne, Melbourne, VIC.

5 Turning Point Alcohol and Drug Centre, Melbourne, VIC.

6 Eastern Health, Melbourne, VIC.

7 Centre for Population Health, Macfarlane Burnet Institute for Medical Research and Public Health, Melbourne, VIC.

Correspondence: t.gibbie@alfred.org.au 


\section{REFERENCES}

1 Loranger A, Janca A, Sartorius N. Assessment and diagnosis of personality disorders. The ICD-10 International Personality Disorder Examination (IPDE). Cambridge, UK: Cambridge University Press, 1997.

2 American Psychiatric Association. Diagnostic and Statistical Manual of Mental Disorders. 4th ed. Text revision. Washington, DC: APA, 2000.

3 Jackson $H$, Burgess P. Personality disorders in the community: a report from the Australian National Survey of Mental Health and Wellbeing. Soc Psychiatry Psychiatr Epidemiol 2000; 35: $531-538$.

4 Ross J, Teesson M, Darke S, et al. The characteristics of heroin users entering treatment: findings from the Australian Treatment Outcome Study (ATOS). Drug Alcohol Rev 2005; 24: 411-418.

5 Brooner R, King V, Kidorf $M$, et al. Psychiatric and substance use comorbidity among treatment-seeking opioid abusers. Arch Gen Psychiatry 1997; 54: 71-80.

6 Khantzian E, Treece C. DSM-III psychiatric diagnosis of narcotic addicts. Arch Gen Psychiatry 1985; 42: 1067-1071.

7 Rounsaville B, Weissman M, Crits-Christoph K, et al. Diagnosis and symptoms of depression in opiate addicts: course and relationship to treatment outcome. Arch Gen Psychiatry 1982; 39: 151-156.
8 Rounsaville B, Weissman M, Kleber H, Wilber C. Heterogeneity of psychiatric diagnosis in treated opiate addicts. Arch Gen Psychiatry 1982; 39: 161-166.

9 Nace E, Davis C, Gaspari J. Axis II comorbidity in substance abusers. Am J Psychiatry 1991; 148: 118-120.

10 Darke S, Williamson A, Ross J, et al. Borderline personality disorder, antisocial personality disorder and risk-taking among heroin users: findings from the Australian Treatment Outcome Study (ATOS). Drug Alcohol Depend 2004; 74 : 77-83.

11 Kidorf M, Disney E, King V, et al. Prevalence of psychiatric and substance use disorders in opioid abusers in a community syringe exchange program. Drug Alcohol Depend 2004; 74: 115122.

12 Hides L, Lubman DI, Devlin H, et al. The Reliability and Validity of $\mathrm{K} 10$ and $\mathrm{PHO}$ amongst injecting drug users. Aust N Z J Psychiatry 2007; 41: 166-168.

13 World Health Organization. International classification of diseases and related health problems. 10th revision. 2nd ed. Geneva: WHO, 1992.

14 Lewin TJ, Slade T, Andrews G, et al. Assessing personality disorders in a national mental health survey. Soc Psychiatry Psychiatr Epidemiol 2005; 40: 87-98.
15 Sheehan D, Janavs J, Baker R, et al. MINI. Mini International Neuropsychiatric Interview. English Version 5.0.0. DSM-IV. Tampa, Fla: Sheehan DV and Lecrubier Y, 2006.

16 Lecrubier Y, Sheehan D, Weiller E, et al. The Mini International Neuropsychiatric Interview (MINI). A short diagnostic structured interview: reliability and validity according to the CIDI. Eur Psychiatry 1997; 12: 224-231.

17 Kessler RC, Andrews G, Colpe LJ, et al. Short screening scales to monitor population prevalences and trends in non-specific psychological distress. Psychol Med 2002; 32: 959-976.

18 Andrews G, Slade T. Interpreting scores on the Kessler Psychological Distress Scale (K10). Aust N Z J Public Health 2001; 25: 494-497.

19 Group WAW. The Alcohol, Smoking and Substance Involvement Screening Test (ASSIST): development, reliability and feasibility. Addiction 2002; 97: 1183-1194.

20 Hameniuk R, Ali R, Babor TF, et al. Validation of the alcohol, smoking, and substance involvement screening test (ASSIST). Addiction 2008; 103: 1039-1047.

21 Murphy B, Herrman H, Hawthorne G, et al. Australian WHOQOL instruments: user's manual and interpretation guide. Melbourne: Australian WHOQOL Field Study Centre, 2000.

(Received 23 Aug 2010, accepted 18 Apr 2011). 